\title{
Charmonium Production at Tevatron, HERA and LHC *
}

\author{
M. A. Sanchis-Lozano ${ }^{a, b \dagger}$ \\ (a) Departamento de Física Teórica \\ (b) Instituto de Física Corpuscular (IFIC) \\ Centro Mixto Universitat de València-CSIC \\ Dr. Moliner 50, E-46100 Burjassot, Valencia (Spain)
}

\begin{abstract}
Charmonia hadro- and photo-production are analyzed in the framework of the color-octet model taking into account higher-order effects induced by initial-state radiation of gluons. We argue that color-octet matrix elements obtained from Tevatron data might be reconciled with those extracted from HERA data on inelastic $J / \psi$ photoproduction. Finally we estimate cross sections for events with muons from prompt $J / \psi$ 's as a background for the $B$ physics programme at LHC experiments.
\end{abstract}

\section{INTRODUCTION}

Over the last years, charmonium physics has recovered a leading role in the challenge of understanding both perturbative and non-perturbative aspects of the strong interaction dynamics. The surplus of $J / \psi$ and $\psi^{\prime}$ resonance production found at the Fermilab Tevatron [1] triggered an intense theoretical activity as conventional wisdom (i.e. the color-singlet model) failed dramatically to reproduce experimental data. Such a discrepancy, by more than an order of magnitude, led to the introduction by Braaten and Fleming [2] of a new production mechanism, the so-called coloroctet model (COM), later cast into a well defined framework based on an effective non-relativistic field theory (NRQCD) [3]. However, although those non-perturbative parameters introduced in the model and needed to bring accordance with experimental data can be rigorously defined in the effective theory (as vacuum expectation values of effective field operators), the possibility of obtaining numerical values from lattice calculations seems unfortunately still far. This current theoretical inability means in practice that such long-distance parameters have to be determined from fits to experimental data.

\footnotetext{
*Work partially supported by CICYT under contract AEN-96/1718 and by an Acción Especial de la Generalitat Valenciana, code 6223080

†E-mail: mas@evalvx.ific.uv.es
}

On the other hand, there is the founded conviction that due to the distinct energy scales, charmonium production cross section at high transverse momentum factorizes into a short- and a long-distance part, the former calculable from perturbative $\mathrm{QCD}$, the latter parameterized by NRQCD matrix elements expected to be universal - i.e. the same for different production mechanisms and energies. However, numerical values of some non-perturbative parameters extracted from Tevatron [4] and HERA [5] apparently are in disagreement each other, casting some doubts on the validity of the COM itself, or at least on the above-mentioned universality hypothesis.

This paper is partially devoted to argue that the claim on the inconsistency of the NRQCD matrix elements obtained from hadro- and photoproduction may be premature because the effective intrinsic transverse momentum of partons has been overlooked.

Indeed, it was well known long time ago that particle production in hadron-hadron collisions requires the introduction, usually as a phenomenological input, of a primordial $k_{t}$ of partons in addition to their expected Fermi motion [6]. During this Conference, we have heard Gemme and Lazzeroni discuss the need of a large intrinsic $k_{t}$ to reproduce experimental data on $D$ and $B$ production. In fact, most of the effective transverse momentum of partons should be attributed 
to multi-gluon emission by the initial state. In the literature sometimes this effect is simulated or simply parameterized by means of a convolution of the hard process cross section with a Gaussian $k_{t}$ distribution whose width is an adjustable parameter [7]. However, strictly speaking the effect is modeled more reliably by using a Monte Carlo generation of the real complexity of the initialstate radiation. As in our previous analysis of charmonium hadro-production [8] we have used PYTHIA [9] managing multi-gluon emission according to an elaborated algorithm [10].

\section{EFFECT OF THE INITIAL RADIA- TION ON THE $p_{t}$ DISTRIBUTION}

Let us point out that, technically, the inclusion of multi-gluon emission by itself hardly changes the overall cross section for charmonium production obtained from PYTHIA. However, the smearing caused by the initial-state radiation is responsible for a migration of events towards the high- $p_{t}$ tail of the charmonium distribution, thus enhancing the differential cross section in the region where experimental points actually lie. In figure 1 (see Ref. [11]) this effect is shown, clearly pointing out the need for a correction of the results from Cho and Leibovich [4] if a more realistic description of the underlying hadronic complexity of the interaction (i.e. initial-state radiation) is required.

In taking into account this $p_{t}$-dependent $K$ factor, new (lower) values for the long-distance parameters, and ultimately the color-octet matrix elements, are obtained from charmonium hadroproduction. In Table 1 we present our values [11 determined from older Tevatron data [1] together with the new ones 12,13 on $J / \psi$ hadroproduction, both showing a better agreement 13] with HERA results than before [4, 13.

\section{THEORETICAL UNCERTAINTIES}

There is no space to review all the theoretical uncertainties related to the extraction of matrix elements from charmonium production which on the other hand have extensively been reviewed in the literature (see for example [14]). Let us only

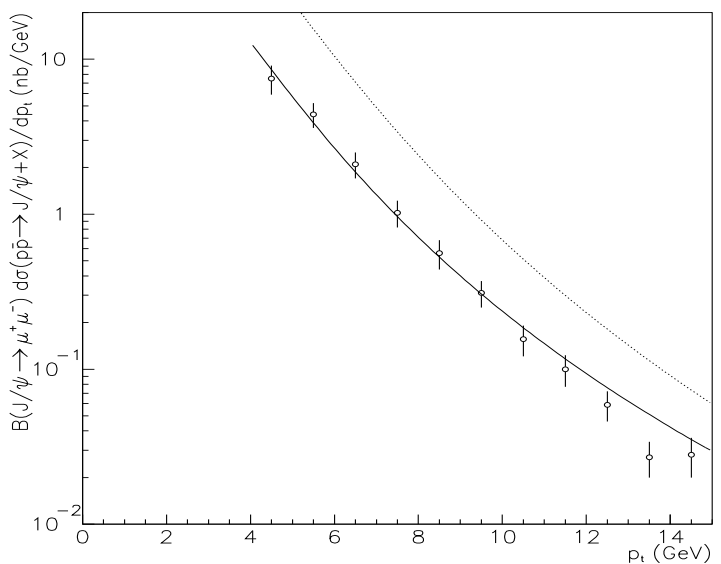

Figure 1. Curves obtained from PYTHIA (not fit) including the color-octet mechanism for $J / \psi$ production at the Tevatron using the same parameters and parton distribution function as in Ref. [4]. The solid line corresponds to initial and final state radiation off and the dotted line to initial and final state radiation on.

mention:

- Ambiguities in the choice of energy scales, charm mass, PDF, ...

- Partial breaking of the heavy-quark spin symmetry in charmonia systems, ...

- Subleading $O\left(>v^{7}\right)$ contributions, higherorder $\alpha_{s}$ effects, ...

- Possible kinematic effects near boundaries of phase space

- Possible effects due to the surrounding medium breaking universality

Below we comment in some detail on the possible effects due to the sensitivity to the kinematics of soft gluon radiation in the long-distance evolution of the $c \bar{c}$ pair into final charmonium 15. 
Table 1

Color-octet matrix elements (in units of $\mathrm{GeV}^{3}$ ) from best fits to old and new Tevatron data on prompt $\underline{J / \psi \text { production. }\left(M_{r}^{J / \psi}=<0\left|O_{8}^{J / \psi}\left({ }^{1} S_{0}\right)\right| 0>+r \cdot<0\left|O_{8}^{J / \psi}\left({ }^{3} P_{0}\right)\right| 0>/ m_{c}^{2} \cdot\right)}$

\begin{tabular}{|c|c|c|}
\hline NRQCD matrix element: & $<0\left|O_{8}^{J / \psi}\left({ }^{3} S_{1}\right)\right| 0>$ & $M_{r}^{J / \psi}$ \\
\hline Ref. 11 (GRV, $r=3$ ): & $(3.4 \pm 0.4) \times 10^{-3}$ & $(6.0 \pm 1.2) \times 10^{-3}$ \\
\hline Ref. 13 ( $(r=3.5)$ : & $(2.73 \pm 0.45) \times 10^{-3}$ & $(5.72 \pm 1.84) \times 10^{-3}$ \\
\hline
\end{tabular}

\subsection{Finite width effects}

Finite width effects can have consequences as Ernström, Lönnblad and Vänttinen discussed some time ago [16]. Indeed, even a small energy difference (of order $M v^{2}$ ) between the mass of final charmonium and the invariant mass of the created $c \bar{c}$ pair should have an effect on the (steep) momentum distribution. Nevertheless, the point is that the formation of an intermediate colored $c \bar{c}$ state does not preclude the possibility of an energy deficit and not necessarily an excess w.r.t. charmonium mass, the latter implying the emission of soft gluons as usually considered. In other words, the $c \bar{c}$ pair could get energy from the surrounding medium.

This viewpoint is in fact not surprising since in the color evaporation model [17] based on duality arguments, the cross section for charmonium production is written as an integral over the $\hat{s}$ range $4 m_{c}^{2}$ and $4 m_{D}^{2}$ (i.e. the upper limit equal to open charm production). However, charmonium production takes place in a "hot" medium as Hoyer pointed out in his talk (for more details see Ref. 18), so sub-thresholds effects likely should have an influence. In the improved color evaporation model this means that the heavy quark pair can get energy from the surrounding color field to give open charm production beneath $\hat{s}=4 m_{D}^{2}$ [19].

Analogously in the COM, those dipole transitions occurring during the evolution into final particles should be associated to absorption of soft gluons from the neighborhood as well. For example a $c \bar{c}\left[{ }^{3} S_{1}^{(8)}\right]$ pair may absorb two nonperturbative gluons becoming a $O\left(v^{2}\right)$ Fock component of the $J / \psi$ state or may radiate two soft gluons thus with nearly unity overlap with the $J / \psi$ wave function. The conclusion is that on the average one should consider both possibili- ties, likely decreasing the net impact of the abovementioned effect on the $p_{t}$ distribution.

\section{LHC PREDICTIONS}

As outlined in the previous section, the extraction of NRQCD matrix elements from charmonium production is not yet fully satisfactory due to several theoretical uncertainties. Still more analysis has to be done to check the universality hypothesis allowing comparisons between different production reactions. Polarization of charmonium from gluon fragmentation at high $p_{t}$ might be decisive to verify the validity of the COM [20].

Nevertheless, under the assumption of the validity of the COM to explain charmonia production, long-distance matrix elements obtained from Tevatron data should parameterize those uncertainties to a large extent. Therefore, in extrapolating to LHC energies, predictions should be reliable if correctly performed. In our case, this actually means running PYTHIA with the same parameters and options (e.g. radiation on) as previously employed in the fit to Tevatron experimental points.

In Figure 2 we show our predictions for prompt $J / \psi$ direct production at the LHC using the CTEQ PDF. A caveat is in order, however. In changing the choice for the PDF there are noticeable changes on the production rate by about $50 \%$ in certain regions of $p_{t}$ (see Ref.[11]).

\subsection{Muon cross sections from prompt $J / \psi$ production at the LHC}

We have estimated the cross sections for events with muons from prompt $J / \psi$ 's, passing typical pseudorapidity and transverse momentum cuts, foreseen in the first and second level triggers for $B$ physics at the two LHC experiments ATLAS and 


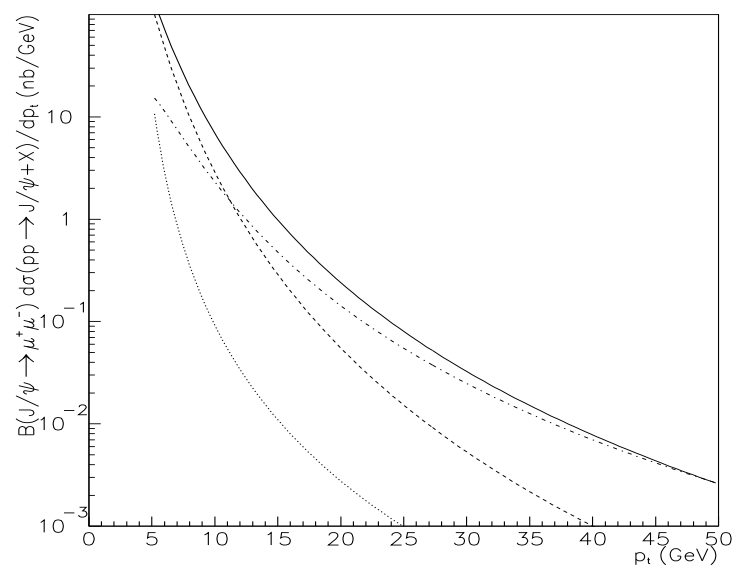

Figure 2. Our predictions for prompt $J / \psi$ direct production at the LHC according to the coloroctet model implemented in PYTHIA for the CTEQ PDF [10]. Dotted line: CSM; dashed line: ${ }^{1} S_{0}^{(8)}+{ }^{3} P_{J}^{(8)}$ contributions; dot-dash line: ${ }^{3} S_{1}^{(8)}$ contribution; solid line: all contributions. Notice that asymptotically the ${ }^{3} S_{1}^{(8)}$ contribution becomes dominant, likely implying a transverse polarization of charmonium at high $p_{t}$.

CMS. We shall denote as $\mu 3, \mu 6(\mu 3 \mu 3, \mu 6 \mu 3)$ those events with at least one (two) muon(s) whose $p_{t}$ is (are) larger than $3 \mathrm{GeV}$ or $6 \mathrm{GeV}$, respectively.

From our modified version of PYTHIA with the COM implemented in, we obtain

$$
\begin{array}{rl}
\sigma(\mu 3)=622 & n b \\
\sigma(\mu 6)=52 & n b \\
\sigma(\mu 3 \mu 3)=30 & n b \\
\sigma(\mu 6 \mu 3)=9 & n b
\end{array}
$$

In all cases an additional pseudorapidity cut $|\eta|<2.5$ for muons was required on generated events.

Those numbers should be useful to evaluate an order-of-magnitude estimate of background sources for the $B$ physics programme at LHC experiments, especially regarding the search for $\mathrm{CP}$ violation via the golden decay mode $B_{d}^{0} \rightarrow J / \psi K_{s}$.

\section{Acknowledgments}

I thank P. Eerola, N. Ellis, P. Hoyer and M. Smizanska for interesting discussions.

\section{REFERENCES}

1. CDF Collaboration, F. Abe at al., Phys. Rev. Lett. 69 (1992) 3704; 71 (1993) 2537; 75 (1995) 1451.

2. E. Braaten and S. Fleming, Phys. Rev. Lett. 74 (1995) 3327.

3. G.T. Bodwin, E. Braaten, G.P. Lepage, Phys. Rev. D 51 (1995) 1125.

4. P. Cho and A.K. Leibovich, Phys. Rev. D 53 (1996) 6203.

5. M. Cacciari and M. Krämer, Phys. Rev. Lett. 76 (1996) 4128.

6. W.M. Geist at al, Phys. Rep. 197 (1990) 263.

7. K. Sridhar, A.D. Martin, W.J. Stirling, DTP98-30, hep-ph/9806253.

8. M.A. Sanchis-Lozano and B. Cano-Coloma, Nucl. Phys. B (Proc. Suppl.) 55A (1997) 277.

9. T. Sjöstrand, Comp. Phys. Comm. 82 (1994) 74.

10. T. Sjöstrand, Phys. Lett. B 157 (1985) 321.

11. B. Cano and M.A. Sanchis-Lozano, Nucl. Phys. B 508 (1997) 753.

12. CDF Collaboration, F. Abe at al., Phys. Rev. Lett. 79 (1997) 578.

13. B.A. Kniehl and G. Kramer, DESY 98-023, hep-ph/9803256.

14. G. Schuler, CERN preprint CERN-TH/98132, hep-ph/9804349.

15. M. Beneke, I.Z. Rothstein and M.B. Wise, Phys. Lett. B 408 (1997) 373.

16. P. Ernström. L. Lönnblad, M. Vänttinen, Z. Phys. C 76 (1997) 515.

17. H. Fritzsch, Phys. Lett. B67 (1977) 217.

18. P. Hoyer and S. Peigne, NORDITA-98/43 HE, hep-ph/9806424.

19. R. Gavai et al., Int. J. Mod. Phys. A 10 (1995) 3043.

20. M. Beneke, CERN preprint CERN-TH/9755, hep-ph/9703429. 